\title{
Reply to 'Reconsidering the Shockley-Queisser limit of a ferroelectric insulator device'
}

Spanier et al. reply - We appreciate the opportunity to answer questions about tip electrodes and their effects on measured current, and the validity of the thermalization length $l_{0}$ in estimating efficiency. Kirk and Cardwell challenge our interpretation by proposing ${ }^{1}$ that the carriers might be collected within the whole slab, rather than just over the thermalization length, based on our findings that $25 \mathrm{~nm}$ and $5 \mu \mathrm{m}$ radii tips yielded similar currents. We respond with the following three arguments.

First, we emphasize that our experimental result involving an array of 24 tips supports our claims. Measurements of power conversion efficiency were performed both using individual nanoscale tips and a hexagonal array of 24 equally spaced indium tin oxide nanoscale tip electrodes over a device area of $\approx 1 \times 1 \mu \mathrm{m}^{2}$ connected over a planar area (Fig. 4 and Methods in ref. 2). If the single tip were collecting current over a very large area, then the array of 24 closely spaced tips would collect the same total current as the single tip. However, we find that the multi-tip array collects several times as much current per unit illumination, invalidating this hypothesis and confirming that the optimal distance between tip-electrode neighbours is close to $l_{0}$.

Second, a 5- $\mu$ m-radius tip does not mean that its effective contact area is considerably larger than that of a 25-nm-radius tip. Actually, the effective contact areas are approximately the same according to the Hertzian tip-sample contact model. We consider our tungsten tip of geometric radius $R=5 \mu \mathrm{m}$ in contact with the $\mathrm{BaTiO}_{3}$ crystal, where the plane of contact is modelled as a sphere of infinite radius. Using an effective Young modulus of the tip-surface system $E^{\star}, E^{\star-1}=\left(\left(1-v_{1}\right) / E_{1}\right)+\left(\left(1-v_{2}\right) / E_{2}\right)$, where $E_{1}$ and $E_{2}$ and $v_{1}$ and $v_{2}$ are the Young moduli and Poisson ratios for the tip material (1, tungsten (W) or platinum $(\mathrm{Pt}) ; E_{1, \mathrm{~W}} \approx 411 \mathrm{GPa}, v_{1, \mathrm{~W}} \approx 0.28$, $\left.E_{1, \mathrm{Pt}} \approx 168 \mathrm{GPa}, v_{1, \mathrm{Pt}}=0.39\right)$ and the crystal $\left(2, \mathrm{BaTiO}_{3} ; E_{2} \approx 150-160 \mathrm{GPa}\right.$ (ref. 3) and $v_{2} \approx 0.65$ (ref. 4)). Under load $P$, which results primarily in observable bending deformation of the probe arm, the indentation depth $w_{0}=\left(3 P / 2 E^{\star}\right)(2 / 3) R^{-1 / 3}$ (ref. 5) and the contact radius is $r=\left(w_{0} R\right)^{1 / 2}$. Given the relatively high value of $E_{1}$, tip deformation effects can be neglected. Calculation of the contact radii $r$ shows that for the $R=5 \mu \mathrm{m}$ tungsten tip and applicable range of loading $(10 \mathrm{nN} \leq P \leq 1 \mu \mathrm{N}), r$ is more than two orders of magnitude smaller than the geometric radius. Thus, both the nano- and micrometre-scale radius probes in our experiment have approximately the same effective contact areas, and $r \ll l_{0}$. For $l_{0}=100 \mathrm{~nm}$ and $1 \mu \mathrm{N}$ loading, the larger tip would have a contact radius of $20 \mathrm{~nm}$ and would therefore have a collection radius of $120 \mathrm{~nm}$, and the smaller tip with contact radius $5 \mathrm{~nm}$ would have a collection radius of $105 \mathrm{~nm}$. The expected current ratio $(120)^{2} /(105)^{2} \approx 1.3$, roughly the ratio between $0.40 \mathrm{nA}$ and $0.3 \mathrm{nA}$ (Fig. $2 \mathrm{~b}$ and c, respectively, from ref. 2), further supporting our argument.

Third, the radius of the screening field is determined by the Debye length $l_{\mathrm{D}}$. For $\mathrm{BaTiO}_{3}, l_{\mathrm{D}} \approx 10^{-5} \mathrm{~cm}$, the relative dielectric constant $\varepsilon_{\text {Вто }} \approx 10^{2}$, and the carrier concentration $n_{\text {Вто }} \approx 10^{17} \mathrm{~cm}^{-3}$. In the tip regime, the open-circuit voltage, $V_{\mathrm{oc}} \approx 8 \mathrm{~V}$ under 3.06-eV-photon illumination, but $V_{\text {oc }}$ can be much less than the energy gap (for example, we find $V_{\text {oc }} \approx 1.2 \mathrm{~V}$ under AM1.5G illumination) because the active length is limited by the free path of non-thermalized electrons $l_{0}$ (and by the Debye length $l_{\mathrm{D}}$ ), which we estimated theoretically to be $\approx 100 \mathrm{~nm}$. We recently reported on the finite-size scaling of the bulk photovoltaic effect, presenting for the first time an experimental determination of the ballistic free path of non-thermalized photogenerated carriers in a ferroelectric. Our results showed that in $\mathrm{BaTiO}_{3}$, at room temperature, the collection length is indeed $l_{0} \approx l_{\mathrm{D}} \approx 100 \mathrm{~nm}$ (ref. 6). Thus, contrary to the suggestion by Kirk and Cardwell that "the carrier collection area may be much larger than the contact area between the sample and the probe tip, and may be as large as the entire $5 \mathrm{~mm} \times 5 \mathrm{~mm}$ sample area itself," our observation of the current collected through an array of tips being many times the single-tip current, the similar effective contact areas of our $25 \mathrm{~nm}$ and $5 \mu \mathrm{m}$ probes, and our recent experimental determination of $l_{0}$, taken together, validate our interpretation that the current is collected from a nanosized hemisphere with radius $l_{0} \approx l_{\mathrm{D}} \approx 100 \mathrm{~nm}$, of area $2 \pi l_{0}^{2}$.

Finally, we point out that in our hypothesis and according to our results, our observed phenomenon does not follow the principle of detailed balance. For a steadystate open circuit, the large depolarization field near the surface (as in our proposed possible mechanism) still exists. This field induces a high non-equilibrium concentration of carriers (excited electrons) near the surface, even though there is a steady-state open-circuit condition. We hope that our laboratory-scale results, analyses and recent experimental findings will stimulate interest in these effects and in efforts to produce and study much largerplanar-area, nanoscale tip electrode arrayed, bulk photovoltaic effect-based cells.

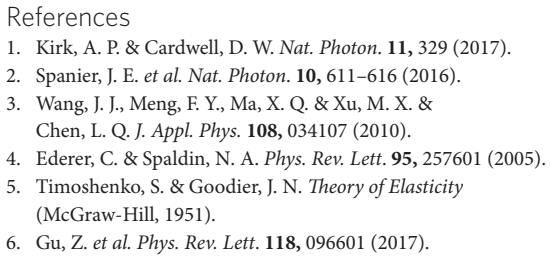

Jonathan E.Spanier ${ }^{1 \star}$, Vladimir M. Fridkin ${ }^{1,2}$, Andrew M.Rappe ${ }^{3}$, Andrew R. Akbashev', Alessia Polemi', Yubo $\mathrm{Qi}^{3}$, Zongquan $\mathrm{Gu}^{1}$, Steve M. Young ${ }^{4}$, Christopher J. Hawley', Dominic Imbrenda', Geoffrey Xiao', Andrew L. Bennett-Jackson ${ }^{1}$ and Craig L. Johnson ${ }^{1}$ 'Drexel University, Philadelphia, Pennsylvania 19104-2875, USA. ${ }^{2}$ Shubnikov Institute for Crystallography, Russian Academy of Sciences, Moscow 117333, Russian Federation. ${ }^{3}$ University of Pennsylvania, Philadelphia, Pennsylvania 19104-6323, USA. ${ }^{4}$ US Naval Research Laboratory, Washington DC 20375, USA. *e-mail:spanier@drexel.edu 